\title{
Comparison of plasma data from ASPERA-3/Mars-Express with a 3-D hybrid simulation
}

\author{
A. Bößwetter ${ }^{1}$, S. Simon ${ }^{1}$, T. Bagdonat ${ }^{1}$, U. Motschmann ${ }^{1,2}$, M. Fränz ${ }^{3}$, E. Roussos ${ }^{3}$, N. Krupp ${ }^{3}$, J. Woch ${ }^{3}$, J. Schüle ${ }^{4}$, \\ S. Barabash ${ }^{5}$, and R. Lundin ${ }^{5}$ \\ ${ }^{1}$ Institute for Theoretical Physics, TU Braunschweig, Germany \\ ${ }^{2}$ DLR, Institute of Planetary Research, Berlin, Germany \\ ${ }^{3}$ Max Planck Institute for Solar System Research, Katlenburg-Lindau, Germany \\ ${ }^{4}$ Institute for Scientific Computing, TU Braunschweig, Germany \\ ${ }^{5}$ Swedish Institute of Space Physics, Kiruna, Sweden
}

Received: 27 July 2006 - Revised: 27 June 2007 - Accepted: 23 July 2007 - Published: 29 August 2007

\begin{abstract}
The ELS and IMA sensors of the ASPERA-3 experiment onboard of Mars-Express (MEX) can measure electron as well as ion moments. We compare these measurements for a specific orbit with the simulation results from a 3-D hybrid model. In the hybrid approximation the electrons are modeled as a massless charge-neutralizing fluid, whereas the ions are treated as individual particles. This approach allows gyroradius effects to be included in our model calculations of the Martian plasma environment because the gyroradii of the solar wind protons are in the range of several hundred kilometers and therefore comparable with the characteristic scales of the subsolar ionospheric interaction region. The position of both the bow shock and the Ion Composition Boundary (ICB) manifest in the MEX data as well as in the results from the hybrid simulation nearly at the same location. The characteristic features of these boundaries, i.e. an increase of proton density and temperature at the Bow Shock and a transition from solar wind to ionospheric particles at the ICB, are clearly identifiable in the data.
\end{abstract}

Keywords. Interplanetary physics (Planetary bow shocks) Space plasma physics (Kinetic and MHD theory; Numerical simulation studies)

\section{Introduction}

Mars does not possess a global intrinsic magnetic field. Therefore, the solar wind can interact directly with the ionosphere of the planet. This interaction leads to an erosion of the Martian atmosphere by different processes: ion pickup, sputtering as well as ionospheric outflow by momentum

Correspondence to: A. Bößwetter

(a.boesswetter@tu-bs.de) transport of solar wind and by detached ionospheric plasma clouds. The first who estimated the atmospheric loss rates were Lundin et al. (1990) using ASPERA measurements from Phobos-2.

The measurements carried out by two spacecraft Phobos2 and Mars Global Surveyor (MGS) led to an improvement of our knowledge about the regions and plasma boundaries surrounding Mars. The Martian plasma environment can be characterized by a set of sharply pronounced plasma boundaries, whose major features are listed in the following paragraphs.

- Due to the supersonic nature of the solar wind, the interaction gives rise to a bow shock. The major features of the bow shock have proven to be completely reproducible in the framework of gasdynamic, magnetohydrodynamic and hybrid models (Spreiter and Stahara, 1992; Liu et al., 1999; Brecht, 1997). The thickness of the magnetosheath, following the bow shock, is of the order of the solar wind proton gyroradius (hundreds of $\mathrm{km}$ ), but thinner than the gyroradius of heavy ions (thousands of $\mathrm{km}$ ) from the planet. Mass-loading of the shocked solar wind by planetary heavy ions occurs within the magnetosheath, indicating the existence of an extended hydrogen and oxygen exosphere. Hybrid simulations for unmagnetised planets (Shimazu, 2001; Terada et al., 2002; Kallio and Janhunen, 2002; Bößwetter et al., 2004; Modolo et al., 2005), a statistical analysis of data from Pioneer Venus Orbiter (Kanao et al., 2006) as well as data from MGS (Vennerstrom et al., 2003) have shown that the direction and strength of the convective solar wind electric field $\boldsymbol{E}_{s w}=-\boldsymbol{V}_{s w} \times \boldsymbol{B}$ plays an important role on the shape of the planetary heavy ion tail, the formation and altitude of plasma boundaries and the magnetic field topology. The convective electric field

Published by Copernicus Publications on behalf of the European Geosciences Union. 
gives rise to pronounced asymmetries in these structures. The gyroradius of the newly generated $\mathrm{O}^{+}$ions significantly exceeds the size of the obstacle. Ions generated upstream of the bow shock have gyroradii of the order of $20000 \mathrm{~km}$; in the magnetosheath the gyroradii become comparable to the magnetosheath width. However, for an analysis of these asymmetries both plasma data and magnetometer data would be necessary. Unfortunalety MEX is not equipped with a magnetometer.

- The Magnetic Pile-up Region (MPR) is a region dominated by planetary ions. A pronounced boundary layer, the Magnetic Pile-up Boundary (MPB), separates the MPR from the magnetosheath. The MPB is a thin, sharp transition region where the solar wind proton density drops sharply (Riedler et al., 1991; Vignes et al., 2000). The magnetometer on Phobos-2 detected this boundary by a rotation of the magnetic field direction and a decrease in turbulence (Riedler et al., 1989). Within the MPR, at the dayside, the solar wind magnetic field piles up and drapes around Mars. At the dayside, the MPR is confined from below by the ionosphere or the exobase, depending on solar wind conditions. At the nightside, the MPR is bounded by the tail region stretching far beyond the planet. In analogy, the MPR and the MPB have also been observed at Venus (Zhang et al., 1991) and comets (Mazelle et al., 1989; Rème et al., 1993), and evidence is emerging that these are common features of the interaction of the solar wind with ionospheres of unmagnetized bodies.

- Further plasma instruments on Phobos-2 also detected boundaries near the location of the MPB (Sauer et al., 1992). They were termed as the magnetopause (Rosenbauer et al., 1989; Lundin et al., 1989), the Protonopause (Sauer et al., 1994), and the Ion Composition Boundary (Breus et al., 1991). The term Ion Composition Boundary (ICB) expresses the separation of the solar wind protons from the planetary ions. It seems that these boundaries are all of the same physical origin.

- MGS instruments measured an other transition region where the energetic electron flux drops abruptly by nearly one order of magnitude (Acuña et al., 1998; Mitchell et al., 2000). This feature gives evidence for the existence of an additional boundary between the MPR and the ionosphere. However, it is still not clear whether this boundary is identical to the ionopause. Several pre-MGS studies, like Hanson and Mantas (1988) analysing Viking measurements, showed that the ionospheric peak thermal pressure at Mars is smaller than the average solar wind ram pressure. Therefore, the ionopause at Mars is not clearly defined as in the case of Venus. In that case, a strong gradient in the cold electron density could be observed at the ionopause
(Luhmann, 1995). At Mars it is expected that magnetic field can penetrate into the Martian ionosphere, much like Venus' ionosphere during times of high solar wind dynamic pressure and low fluxes of ionizing solar radiation. Locally, crustal magnetic fields, occuring mainly in the Southern Hemisphere, give rise to an even more complex topology of the ionosphere and the "ionopause" (Acuña et al., 1998).

ASPERA and TAUS instruments on the Phobos-2 mission allowed the first direct detection of escaping Martian ions (Lundin et al., 1989; Rosenbauer et al., 1989). The interpretation of these measurements leading to both ion pick-up effects in the so-called Martian boundary layer, which corresponds to the MPR, and ionospheric $\mathrm{O}^{+}$beams deeper into the tail.

The finite gyroradius of the solar wind protons is of the order of hundreds of kilometers and is therefore comparable to the characteristic length scales of the interaction region. A hybrid model is capable of describing these kinetic processes. It treats the electrons as a fluid, whereas a completely kinetic description is retained to cover ion dynamics. The hybrid approach is used to study the properties of the plasma and the magnetic field environment near Mars (Brecht, 1997; Shimazu, 1999; Kallio and Janhunen, 2002; Bößwetter et al., 2004; Modolo et al., 2005) and can also applied to other unmagnetised objects such as Titan (Simon et al., 2006a,b) and weak comets (Bagdonat and Motschmann, 2002a,b; Bagdonat et al., 2004). In correspondence to MEX measurements Kallio et al. (2006) was able to identify escaping planetary ions in the hemisphere where the convective electric field points away from Mars. Modolo et al. (2005) studied the Martian plasma environment under influence of different solar EUV fluxes. They concluded that the Martian plasma environment features a pronounced asymmetry with respect to the direction of the convective electric field of the solar wind flow.

Recent in situ measurements of ions and electrons were obtained by the ASPERA-3 instrument onboard MEX coming from the IMA (Ion Mass Analyser) and ELS (Electron Spectrometer) detectors. Fränz et al. (2006a) calculated electron and ion moments from the raw data of these detectors and developed density and temperature maps of the Martian plasma environment. They revealed the boundaries of the Mars-solar wind interaction and gave a qualitative description of the plasma behavior at the different interaction regions.

In this paper, we present IMA and ELS data from a specific orbit and compare it to our hybrid model. After giving a brief description of the hybrid model we show the simulation results for the solar wind, planetary ions and the magnetic field near Mars. Finally, a comparative discussion will be given. 


\section{Observations: Mars Express orbit No. 1614}

The ELS and IMA sensors of the ASPERA-3 Experiment onboard of MEX can measure electron as well as ion moments. In this paper we use electon data from the ELS sensor with a high $4 \mathrm{~s}$ time resolution and ion data from the IMA sensor with $192 \mathrm{~s}$ time resolution. IMA provides ion measurements in the energy range between 0.02 and $30 \mathrm{keV} / \mathrm{q}$ for the main ion components with the charge number $q$. ELS provides electron measurements in the energy range between 0.01 and $20 \mathrm{keV}$. A general description of the instrument is given in Barabash et al. (2006).

We choose orbit No. 1614 on 19 April 2005 as a representive MEX orbit for the first two years between 1 February 2004 and 1 February 2006. The selection of the orbit is not only because solar wind conditions look normal, also because the specific orbit does not cross over regions of strong crustal fields allowing a analysis with the interaction of magnetic field which is induced by the solar wind. As the simulation code does not include the model for the crustal field network, this selection is appropriate.

Figure 1 depicts the position of MEX on this orbit between 15:15 UT and 17:40 UT. As to be seen in Fig. 1a, the orbital plane lies more in the polar (x-z plane) than in the equatorial plane (y-x plane) of Mars. The spacecraft crosses the bow shock at 15:25 UT, here shown as projections of MGS measurements (Vignes et al., 2000) into the cutting planes of our simulation coordinate system, from the dusk side of the planet. After reaching a closest approach altitude of $330 \mathrm{~km}$ in the north pole region at 16:51 UT MEX leaves the magnetosheath again near the dayside region at about 17:25 UT. Figure 1c shows a view from the night side of the planet.

As can be seen from the following altitudes of the trajectory, the spacecraft surrounded the planet on a highly elliptic orbit: 16.00 at $2.3 R_{M}(4420 \mathrm{~km}), 16: 30 \mathrm{UT}$ at $1.5 R_{M}$ $(1700 \mathrm{~km})$ and 17:00 UT at $1.1 R_{M}(340 \mathrm{~km})$.

The energy spectra of electrons and ions are displayed in Figs. $2 \mathrm{a}$ and $\mathrm{b}$, respectively. The two bow shock crossings are clearly identifiable in the time evolution of the average particle energy along the spacecraft trajectory. As denoted in the figure, between 15:55 UT and 17:08 UT, the spacecraft passes a region of cold plasma with a electron temperature of $10 \mathrm{eV}$. Ionospheric plasma is much colder; this may be accelerated plasma, but not typical ionospheric population. Nevertheless, in the same region, the spacecraft detects only minor concentrations of cold ions of ionospheric origin. Significant densities of heavy ions occur only at the flanks of a signature which we will interpret later as MPB, their energy being of the order of $400 \mathrm{eV}$. This can clearly be seen in Fig. $2 b$, denoted by the green regions below $1 \mathrm{keV}$.

Between 16:30 UT and 16:50 UT, the instrument detects significant fluctuations in the electron energy spectrum. According to Fränz et al. (2006b), these distorsions can be ascribed to crustal magnetic fields, affecting the plasma environment in the immediate vicinity of the surface. Besides,
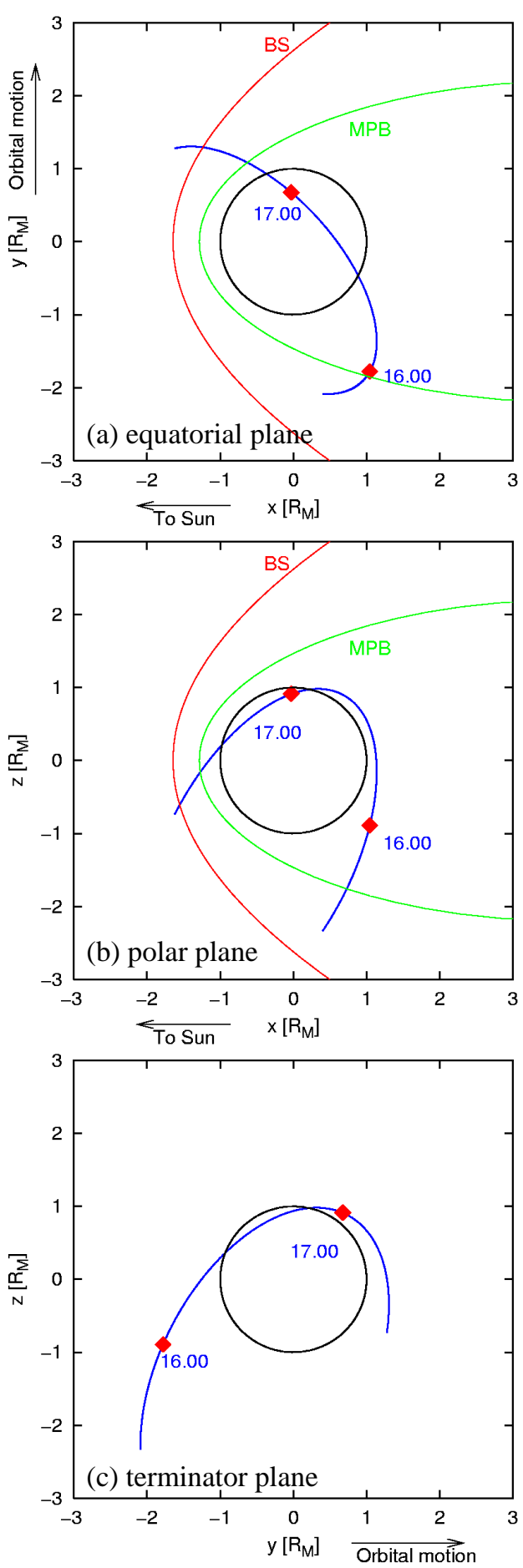

Fig. 1. Pojections of the trajectory of Mars Express orbit No. 1614 on the coordinates planes of our 3-D hybrid simulation. The undisturbed solar wind flows in positive $\mathrm{x}$-direction, the interplanetary magnetic field points in positive y-direction. The convective electric field points to the negative $\mathrm{z}$-direction. The orbital plane is tilted predominatly to the polar (x-z plane). The spacecraft cross the bow shock at 15:25 UT and again at 17:25 UT. 


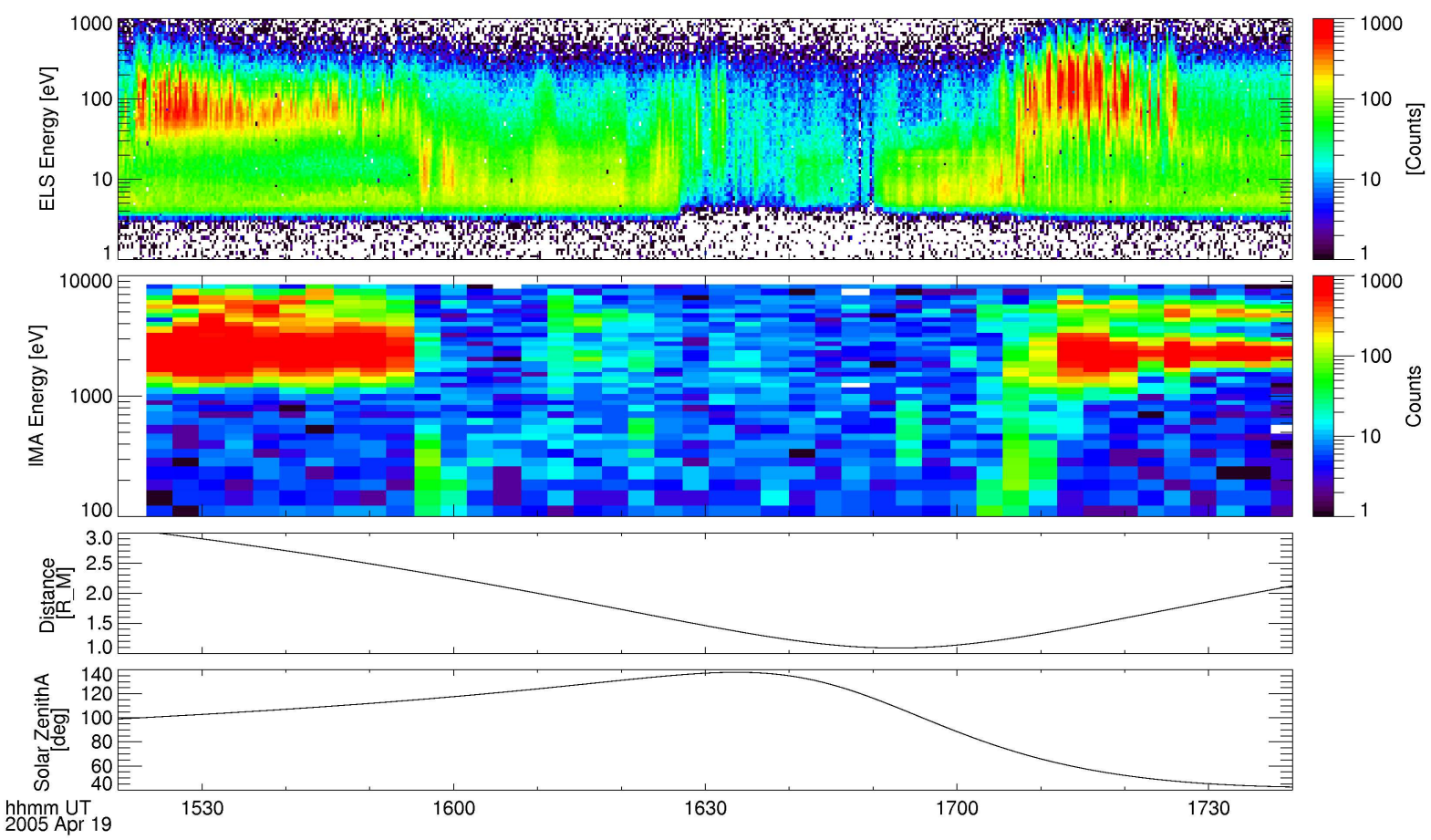

Fig. 2. Mars Express energy spectra of electrons (a) and ions (b) for the orbit No. 1614 on 19 April 2005. The two bow shock crossings appear 15:25 UT and 17:25 UT. Between 15:55 UT and 17:08 UT, the spacecraft pass a region of cold ionospheric plasma. Significant densities of heavy ions occur at the flanks of the MPB/ICB.

in the same time interval, several sharply confined areas of extremely reduced plasma density are detected. As stated by (Brace et al., 1982), such signatures have also proven to be characteristic for the plasma at the nightside of Venus. A more extensive discussion of the plasma signatures detected during the orbit will be given with analysis of Fig. 3 .

In any case, it is important to notice that the quality of the spectra is affected by several experimental factors: On the one hand, the electron spectra are falsified by the electrostatic charging of the spacecraft. However, as the energy resolution of the ELS sensor is sufficiently high to allow an identification of these distorsions in the spectra, the spacecraft charge can be computed and therefore, the spectra can be corrected. Outside of the ionosphere, the spacecraft potential takes different values, the electron moments can be calculated.

On the other hand, even though the IMA instrument is capable of detecting protons in the ionosphere region, it is extremely difficult to discriminate between different heavy ion species, such as molecular and atomic oxygen (cf. Fränz et al., 2006a).

As discussed by Fränz et al. (2006a), moments are calculated by integration of Gaussian fits to the phase space distribution. While integration usually covers the complete energy spectrum, low and high energy parts of the spectrum are fitted separately for distributions outside the ionosphere.
For our comparative study, we use the electron data with a sampling rate of $4 \mathrm{~s}$. In contrast to this, ion data are detected in intervals of 192 s, i.e. compared to the electron spectra, the resolution is quite rough.

Figure 3 shows the variations of the plasma parameters occuring along the trajectory of orbit 1614 . In the solar wind, the electron density is in good quantitative agreement with the proton density, being of the order of 1-3 particles per $\mathrm{cm}^{3}$. The temperatures are also obtained from a fit of the high energy part of the spectrum. In general, the values obtained from the fitting procedure shows to be more representative than the values from an integration of the spectra. Especially, in regions of high proton temperature, the proton temperatures are overestimated by the integration procedure. The data allows to obtain the following set of characteristic solar wind parameters: $T_{e}=3-5 \mathrm{eV}, T_{p}=20-30 \mathrm{eV}, v_{p}=600-$ $700 \mathrm{~km} / \mathrm{s}$ and $P_{t h, e} \approx 0.005$.

Both the electron and the proton data allow a clear identification of the bow shock crossing, as denoted by the green dashed lines in Fig. 3. At the shock ramp, the electron density undergoes a sudden increase from 2 to $8 \mathrm{~cm}^{-3}$, which is in agreement with the Rankine Hugoniot jump conditions for plasma discontinuities. The thermalization of the solar wind plasma goes along with an increase of electron temperature from $5 \mathrm{eV}$ in front of the shock up to $40 \mathrm{eV}$. An analogeous jump from $0.005 \mathrm{nPa}$ to $0.1 \mathrm{nPa}$ manifests in the 
electron pressure. In contrast to this, as the proton temperature as well as their velocity experience only a slight modification, the shock position is not clearly identifiable in the proton data.

At the spacecraft position at 15:55 UT, the IMA instrument detects a significant decrease of the proton velocity from $600 \mathrm{~km} / \mathrm{s}$ to values around $350 \mathrm{~km} / \mathrm{s}$ (blue dashed line). This decrease goes along with a strong reduction of the proton density from $5 \mathrm{~cm}^{-3}$ down to below $1 \mathrm{~cm}^{-3}$. As to be seen from Fig. 2b, this modicfication of the proton density coincides with the first detection of heavy ions by the IMA sensor. This modification of the plasma parameters denote the position of the ICB, preventing the shocked solar wind from mixing with the cold ionospheric plasma flow. This boundary layer is referred to as the MPB, as a pronounced increase of magnetic field strength has also shown to be characteristic for this region. However, since MEX does not have a magnetometer on board, it is impossible to determine whether the decrease of the proton density goes along with changes in the magnetic field topology. Even though the ions are incapable of crossing the ICB (cf. Bößwetter et al., 2004; Simon et al., 2006a,b, for an extensive theoretical discussion), Nagy et al. (2004) suggest that the solar wind electrons are able to cross the ICB/MPB moving along the draped magnetic field lines into ionospheric regimes. Evidence confirming this hypothesis can be found in the spectrum. As displayed in Figs. $2 \mathrm{a}$ and 3 , even at the nightside, local concentrations of electrons possessing a relatively high thermal energy around $10-15 \mathrm{eV}$ are detected. These signatures are encountered between 16:00 UT and 17:00 UT. However, the ELS instrument also detects cold electrons with a temperature of $4 \mathrm{eV}$ where this temperature is determined by an extrapolation to the low energy range regime (here not presented). These particles definitely originate from the planetary ionosphere. Hanson and Mantas (1988) who analyzed Viking data obtained a temperature of $0.5 \mathrm{eV}$ for this electron population, being about a factor of 8 smaller than the value obtained from the MEX spectra. In consequence, it must be assumed that the electron population detected by MEX consists of solar wind as well as of ionospheric electrons. On the other hand, a certain falsification of the temperature values must again be ascribed to the spacecraft potential. Due to the ELS low energy cut-off temperatures below $1 \mathrm{eV}$ cannot be measured.

Between 17:00 UT and 17:08 UT, the IMA sensor detects again heavy ions of planetary origin. The second crossing of the ICB occurs at 17:08 UT and is again denoted by a blue dashed line. At 17:25 UT, the spacecraft leaves the induced magnetosphere of Mars, as indicated by the bow shock crossing (green dashed line). As can be seen in Fig. 1b of the Martian polar plane, MEX crosses the bow shock at different shock positions. This can also be seen from Fig. 3, showing that in the subsolar region (outbound) the shock is more compressed than in the Southern Hemisphere (inbound).

To sum up, the position of both the bow shock and the ICB manifest in the MEX data. The characteristic features of
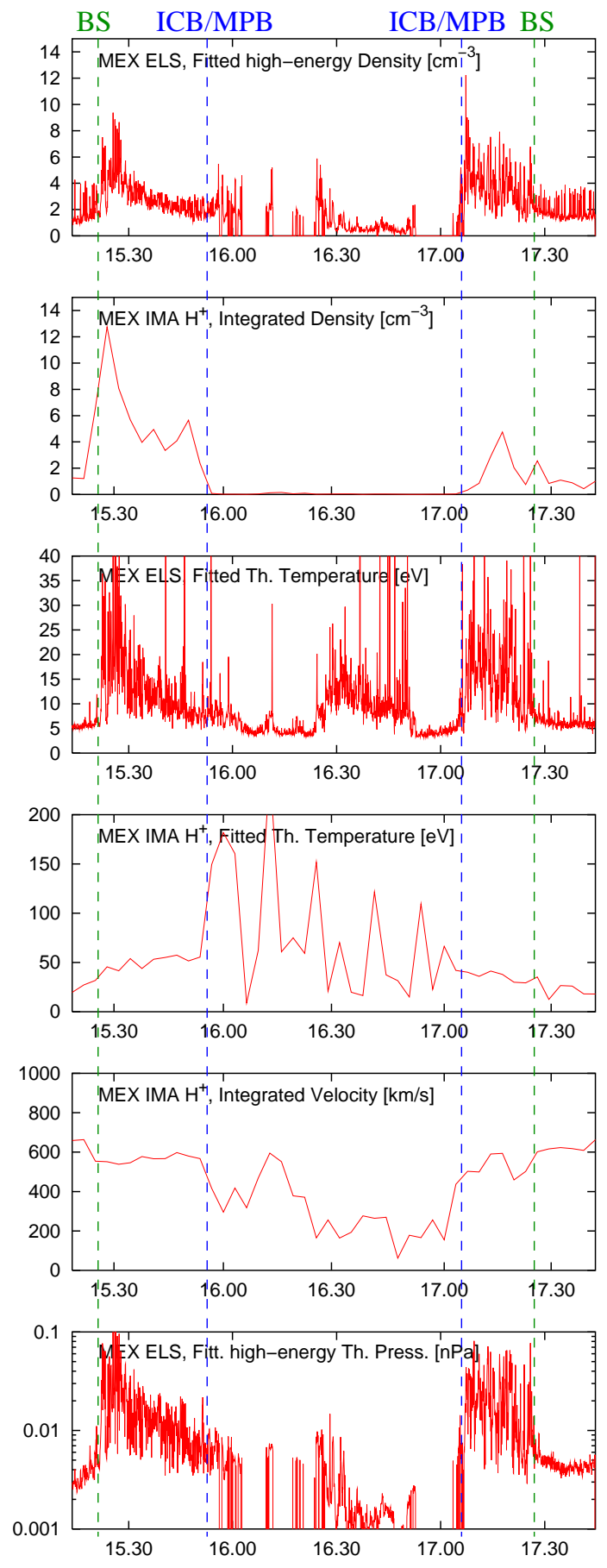

Fig. 3. Mars Express ELS and IMA data. This figure shows from top to bottom: electron density calculated from the high energy part of the spectrum, proton density integrated from the full enery range of the spectrum, electron temperature calculated from a fit to the whole spectrum, proton temperature calculated from a fit to the whole spectrum, integrated proton velocity and thermal electron pressure calculated from the high energy part of the spectrum. 
these boundaries, i.e. an increase of proton density and temperature at the Bow Shock and a transition from solar wind to ionospheric particles at the ICB, are clearly identifiable in the data.

\section{Hybrid model}

The numerical investigations are done using a hybrid code by Bagdonat and Motschmann (2002a). The present version of the code has already been successfully applied to the solar wind interaction with comets (Bagdonat et al., 2004), magnetized asteroids (Simon et al., 2006a) as well as to the plasma environment of Mars (Bößwetter et al., 2004) and Titan (Simon et al., 2006b; Motschmann and Kührt, 2006).

In the hybrid approximation the electrons are modeled as a massless charge-neutralizing fluid, whereas the ions are treated as individual particles. Here we do not dwell into the detailed description of the code. It can be found in Bößwetter et al. (2004). In the following, the dynamic equations of the model will be presented.

- Equation of motion for individual ions:

$$
\frac{\mathrm{d} \boldsymbol{v}_{s}}{\mathrm{~d}}=\frac{q_{s}}{m_{s}}\left(\boldsymbol{E}+\boldsymbol{v}_{s} \times \boldsymbol{B}\right)-k_{D} n_{n}\left(\boldsymbol{v}_{s}-\boldsymbol{u}_{n}\right)
$$

where $q_{s}, m_{s}$ and $\boldsymbol{v}_{s}$ denote the charge, mass and velocity of an individual particle of species $s$, respectively. $k_{D}$ is a phenomenological constant describing the collisions of ions and neutrals, and has been given as $1.7 \times 10^{-9} \mathrm{~cm}^{3} \mathrm{~s}^{-1}$ by Israelevich et al. (1999). $n_{n}$ and $\boldsymbol{u}_{n}$ are the number density and bulk velocity of the neutrals. We use $\boldsymbol{u}_{n}=0$.

- Electric field equation:

$$
\boldsymbol{E}=-\boldsymbol{u}_{i} \times \boldsymbol{B}+\frac{(\nabla \times \boldsymbol{B}) \times \boldsymbol{B}}{\mu_{0} e n_{e}}-\frac{\nabla P_{e, \mathrm{sw}}+\nabla P_{e, \mathrm{hi}}}{e n_{e}}
$$

where $\boldsymbol{u}_{i}$ is the mean ion velocity. Since the plasma is assumed to be quasi-neutral, the mean ion density $\left(n_{i}\right)$ is equal to the electron density $\left(n_{e}\right)$. As the electron temperature in the solar wind differes by several orders of magnitude from the electron temperature in the Martian ionosphere (Hanson and Mantas, 1988), two different electron pressure terms $P_{e, \text { sw }}$ and $P_{e, \text { hi }}$ have been incorporated into the simulation model. Both electron populations are assumed to be adiabatic, i.e.

$$
P_{e, \mathrm{sw}} \propto \beta_{e, \mathrm{sw}} n_{\mathrm{sw}}^{\kappa} \quad \text { and } \quad P_{e, \mathrm{hi}} \propto \beta_{e, \mathrm{hi}} n_{\mathrm{hi}}^{\kappa}
$$

An adiabatic exponent of $\kappa=2$ was used (Bößwetter et al., 2004).
- Magnetic field equation: For the time evolution of the magnetic field one obtains from Faradays law

$$
\frac{\partial \boldsymbol{B}}{\partial t}=\nabla\left(\boldsymbol{u}_{i} \times \boldsymbol{B}\right)-\nabla \times\left[\frac{(\nabla \times \boldsymbol{B}) \times \boldsymbol{B}}{\mu_{0} e n_{e}}\right] .
$$

The electron pressure terms do not occur in this equation, because of $\nabla \times \operatorname{grad} . . .=0$ applied to adiabatic electrons.

The code operates on a curvilinear grid in three spatial dimensions. The simulations are carried out on a so-called "Fisheye Grid" which can be adapted to the spherical geometry of the obstacle. A detailed description of the grid generation can be found in Bößwetter et al. (2004). It also allows an high spatial resolution in the vicinity of the planetary atmosphere.

The Martian atmosphere is modeled as a spherical symmetric gas cloud around Mars consisting of atomic oxygen. The radial density distribution includes an ionospheric exponential profile and an exospheric $1 / r$ profile for the oxygen corona above $500 \mathrm{~km}$. The detailed features of the profile have been presented by Bößwetter et al. (2004). The model based on the assumption of constant solar UV radiation with a photoionisation frequency of oxygen $\nu=2 \times 10^{-7} \mathrm{~s}^{-1}$ for average conditions and $\nu=1 \times 10^{-7} \mathrm{~s}^{-1}$ for solar minimum. This yields a dayside ion production function $q(r, \chi)$ in the form of a Chapman layer. It depends on both the altitude $r$ above the surface and the solar zenith angle $\chi$. The nightside production profile is assumed to be independent of the solar zenith angle. It is set to an altitude-depending value of $q\left(r, \chi=87^{\circ}\right)$. This yields a peak ion production rate of about $10 \%$ of the dayside value.

Any ion hitting the so-called "Inner Boundary" at an altitude of $120 \mathrm{~km}$ above the planetary surface is removed from the simulation. No boundary conditions are imposed on the electromagnetic fields, i.e. the equations for $\boldsymbol{E}$ and $\boldsymbol{B}$ are solved outside as well as inside the obstacle. An artifical inner density is assumed and increased until the steady state is achieved while the simulation proceeds in order to match the surrounding ionospheric heavy ion density as well as to avoid electric fields arising from density gradients.

\section{Simulation results for the orbit 1614}

The specific values of the solar wind density $\left(n_{s w}\right)$ and velocity $\left(u_{s w}\right)$ are not exactly known for this MEX orbit. As we discussed in Sect. 2, different analysis methods for the spectra yield different solar wind backgound values. For the simulation run 1 we chose the same background parameter as we suggested in Bößwetter et al. (2004) for the standard case. A second simulation run is based on input parameter obtained from ELS and IMA undisturbed solar wind parameter. The main input parameters are listed in Table 1 and Table 2. The 
Table 1. Input parameters for simulation run 1.

\begin{tabular}{lll}
\hline Parameter & Symbol & Numerical value \\
\hline Solar wind density & $n_{s w}$ & $4 \mathrm{~cm}^{-3}$ \\
Solar wind velocity & $u_{s w}$ & $327 \mathrm{~km} / \mathrm{s}$ \\
Total dynamic pressure & $M_{p}$ & $0.71 \mathrm{nPa}$ \\
Alfvenic Mach Number & $M_{A}$ & 10 \\
Background magnetic field & $B_{s w}$ & $3 \mathrm{nT} \mathrm{in} \mathrm{y-direction}$ \\
Proton temperature & $T_{p}$ & $50000 \mathrm{~K}=4.3 \mathrm{eV}$ \\
Electron temperature & $T_{e}$ & $200000 \mathrm{~K}=17.3 \mathrm{eV}$ \\
Ionospheric photoionisation freq. & $v$ & $2 \times 10^{-7} \mathrm{~s}-1$ \\
Ionospheric electron temperature & $T_{e, h i}$ & $3000 \mathrm{~K}=0.26 \mathrm{eV}$ \\
\hline
\end{tabular}

Table 2. Input parameters for the simulation run 2.

\begin{tabular}{lll}
\hline Parameter & Symbol & Numerical value \\
\hline Solar wind density & $n_{s w}$ & $1 \mathrm{~cm}^{-3}$ \\
Solar wind velocity & $u_{s w}$ & $611 \mathrm{~km} / \mathrm{s}$ \\
Total dynamic pressure & $M_{p}$ & $0.62 \mathrm{nPa}$ \\
Alfvenic Mach Number & $M_{A}$ & 7 \\
Background magnetic field & $B_{s w}$ & $4 \mathrm{nT}$ southward \\
Proton temperature & $T_{p}$ & $275000 \mathrm{~K}=25 \mathrm{eV}$ \\
Electron temperature & $T_{e}$ & $44000 \mathrm{~K}=4 \mathrm{eV}$ \\
Ionospheric photoionisation freq. & $v$ & $1 \times 10^{-7} \mathrm{~s}-1$ \\
Ionospheric electron temperature & $T_{e, h i}$ & $3000 \mathrm{~K}=0.26 \mathrm{eV}$ \\
\hline
\end{tabular}

input parameters for run 1 provide a total dynamic pressure of

$M_{p}=n_{p} m_{p} v_{p}^{2}=0.71 \mathrm{nPa}$.

This value fits in the pressure balance and reproduces the bow shock just at the observed position. The simulation run 2 uses a slightly lower value of $M_{p}=0.62 \mathrm{nPa}$ which based on a only imprecisely determinable solar wind velocity.

However, due to the lack of magnetic field data, neither the magnetic field nor its direction are available as input parameters for the simulation. The direction of the magnetic field also determines the orientation of the convective electric field $\boldsymbol{E}_{s w}=-\boldsymbol{U}_{s w} \times \boldsymbol{B}_{s w}$, being of major importance for the asymmetric structure of the ionospheric tail and therefore determining the location of the pick-up region. As stated by Bößwetter et al. (2004) and Simon et al. (2006b), the ionospheric tail exhibits a definite preference to expand into the hemisphere where the electric field is directed away from Mars. The existence of these asymmetries has been confirmed by both simulation and measurements (Brecht, 1997; Kallio and Janhunen, 2002; Modolo et al., 2005; Fedorov et al., 2006). For the MEX orbit discussed in this paper, we chose two scenarious. The simulation run 1 the magnetic field is orientated parallel to the $y$-axis, i.e. $B$ is parallel to the equatorial plane and perpendicular to the undisturbed

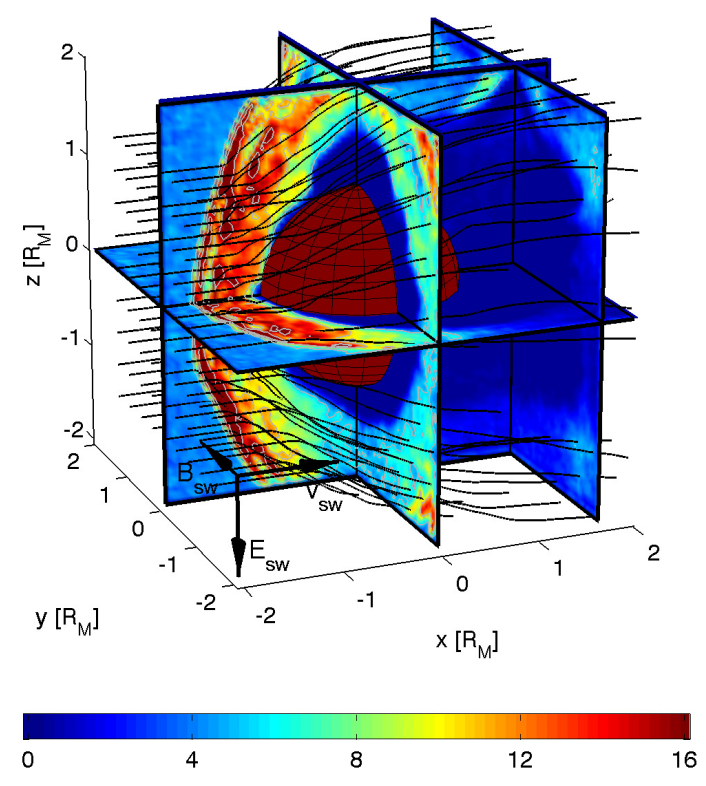

Fig. 4. Simulated solar wind parameters (adaped from Bößwetter et al., 2004). The cutting planes show the solar wind density in $\mathrm{cm}^{-3}$. The black solid lines represent stream lines of the corresponding velocity field.

flow direction. By comparing of MGS upstream dynamic pressure and the direction of the draped magnetic field near Mars D. Brain (private communication, 2007) suggested to use a IMF magnitude of $4 \mathrm{nT}$ and a IMF direction with a mainly southward component. However, these additional parameters were not directly measured in the solar wind regime because at that time MGS was located close to the planet in the draped magnetic field configuration.

The total simulation time is of the order of $1800 \mathrm{~s}$, corresponding to the duration in which the undisturbed solar wind would pass through the entire simulation domain 28 times. Due to the extremely slow motion of the ionospheric particles in the planetary wake, such a long simulation time is absolute mandatory to obtain a quasi-stationary state with a fully developed tail structure. Changing the size of the simulation box from 4 to 6 Martian radii does not effect the results.

The three-dimensional structure of the Martian plasma tail is illustrated in Figs. 4 and 5 by means of global 3-D plots of the solar wind and the heavy ion density. The streaming lines in these figures show the deflection of the plasma flow around the obstacle. The interaction gives rise to a pronounced, cone-shaped cavity of reduced proton density in the wake region, its outer flanks denoting the location of the ICB. As also to be seen in Figs. 4, 5 and 6, the region of reduced proton density is characterized by an increased heavy ion density, i.e. the particles of planetary origin fill the cavity in the proton density. In the following, the hemisphere where the electric field is pointing towards Mars will be refered to as the $E^{-}$hemisphere, whereas the hemisphere where the electric 


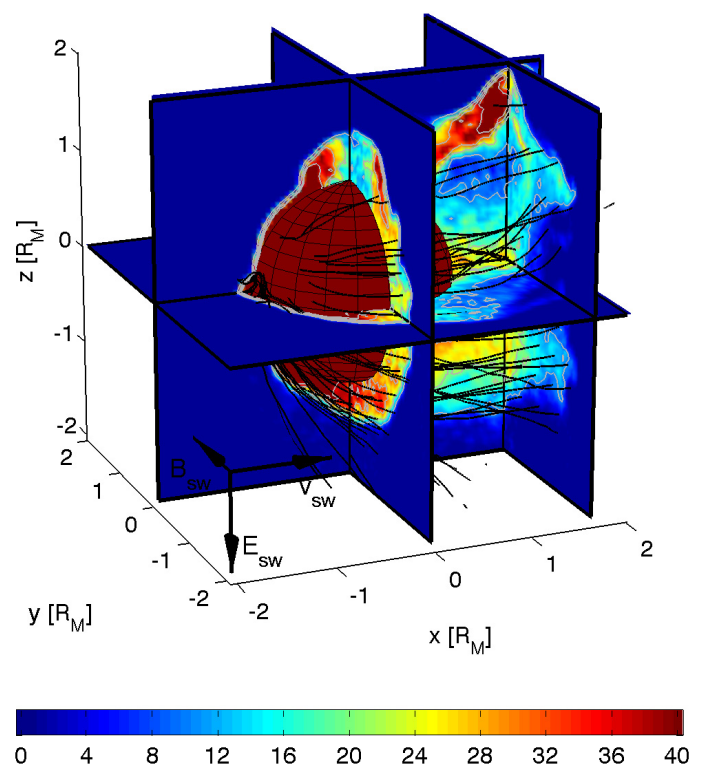

Fig. 5. Simulated ionospheric parameters (adaped from Bößwetter et al., 2004). The cutting planes show the heavy ion density $\left(\mathrm{O}^{+}\right)$ in $\mathrm{cm}^{-3}$. The black solid lines represent the stream lines of the corresponding velocity field. The heavy ions form a complex tail structure behind the planet.

field is directed away from the planet is called the $E^{+}$hemisphere. In other words, the Northern Hemisphere is identical to the $E^{-}$hemisphere, while the Southern Hemisphere coincides with the $E^{+}$hemisphere. On the one hand, in the $E^{-}$hemisphere, the boundary between solar wind and ionospheric ion is sharply pronounced. At the tail's outer flank forms a potential barrier to any heavy ion attempting to leave the tail. In contrast to this, in the $E^{+}$hemisphere, the electric field is directed away from Mars, therefore preventing the formation of a sharphy pronounced boundary layer. An extensive discussion of the underlying meachanism is given by Bößwetter et al. (2004) and in a comparative study by Simon et al. (2007). Due to the proton velocity at the tail's flank in the $E^{-}$hemisphere being about a factor of seven larger than the heavy ion velocity inside the tail, it is likely that a Kelvin-Helmholtz instability is triggered in this region (Penz et al., 2004). The necessity of a kinetic treatment is clearly illustrated by the structure of the bow shock, allowing to identify a set of so-called shocklets. These parabolically shaped regions of enhanced plasma density arise from the proton's finite gyroradius, as has also been discussed by Omidi and Winske (1990); Shimazu (2001) for the case of unmagnetized planets, by Bagdonat and Motschmann (2002b) for the case of weak comets and by Simon et al. (2006a) for the case of magnetized asteroids. A more extensive discussion of the global features of the Martian plasma environment is given by Bößwetter et al. (2004).
For two-dimensional cuts through the simulation box, Figs. 6 and 7 display the solar wind as well as the ionospheric plasma parameters. Both the bow shock and the ICB are clearly identifiable. Besides, as displayed in Fig. 6, the heavy ion density in the equatorial plane exhibits some kind of ray structure at the nightside of the obstacle: The central tail directly beyond the planets is flanked by another signature at either side, being less pronounced than the density enhancement in the central region. Lichtenegger and Dubinin (1998) found a similar tail structure using test particle simulations. In general, the situation in the equatorial plane is highly symmetric, while a pronounced asymmetry with respect to the direction of the convective electric field occurs in the polar plane. The asymmetric structure of the pick-up region seems to be a common feature of the plasma environment of unmagnetized planets. Recently, Fedorov et al. (2006) was able to confirm the existence of such a structure for the Martian scenario. A similar structure could also be detected during the first Cassini flybys of Titan, as discussed by Wahlund et al. (2005). An explanation for this signature was given by Simon et al. (2006b). The strong analogy between the Martian plasma environment and the interaction of Titan with the saturnian magnetospheric plasma was emphasized in a comparative discussion by Simon et al. (2007). These asymmetries should be taken into consideration when analyzing the data from MEX. However, in the situation under consideration this is extremely difficult since their formation depends strongly on the orientation of the magnetic field.

\section{Comparison of the simulation results with MEX orbit}

The results of a comparison between simulation and observation are shown in Figs. 8 and 9 for simulation run 1 and 2 , respectively. The plots show the electron temperature and pressure as well as the components of the proton velocity. The simulation data were extracted for the MEX trajectory, allowing a discussion of the plasma parameters as a function of spacecraft time.

As can be seen in Fig. 8, when entering the induced Martian magnetosphere, the spacecraft is located inside the magnetosheath for a duration of about $30 \mathrm{~min}$, while the outbound pass is significantly shorter. These features are completely reproducible in the framework of the hybrid simulation model. Figure 9 shows the same data and the simulation results for run 2 . In this simulation the inbound crossings through the bow shock and the ICB/MPB appear about 15 min later.

The shocklet structure that could be identified in the simulation results is detected by the ELS instrument at 15:30 UT and 17:20 UT. The density enhancements in the shocklets obtained from the simulations are in good qualitative agreement with the data displayed in Figs. 8 and 9 (right). Minor differences between simulation and measurements can be ascribed 

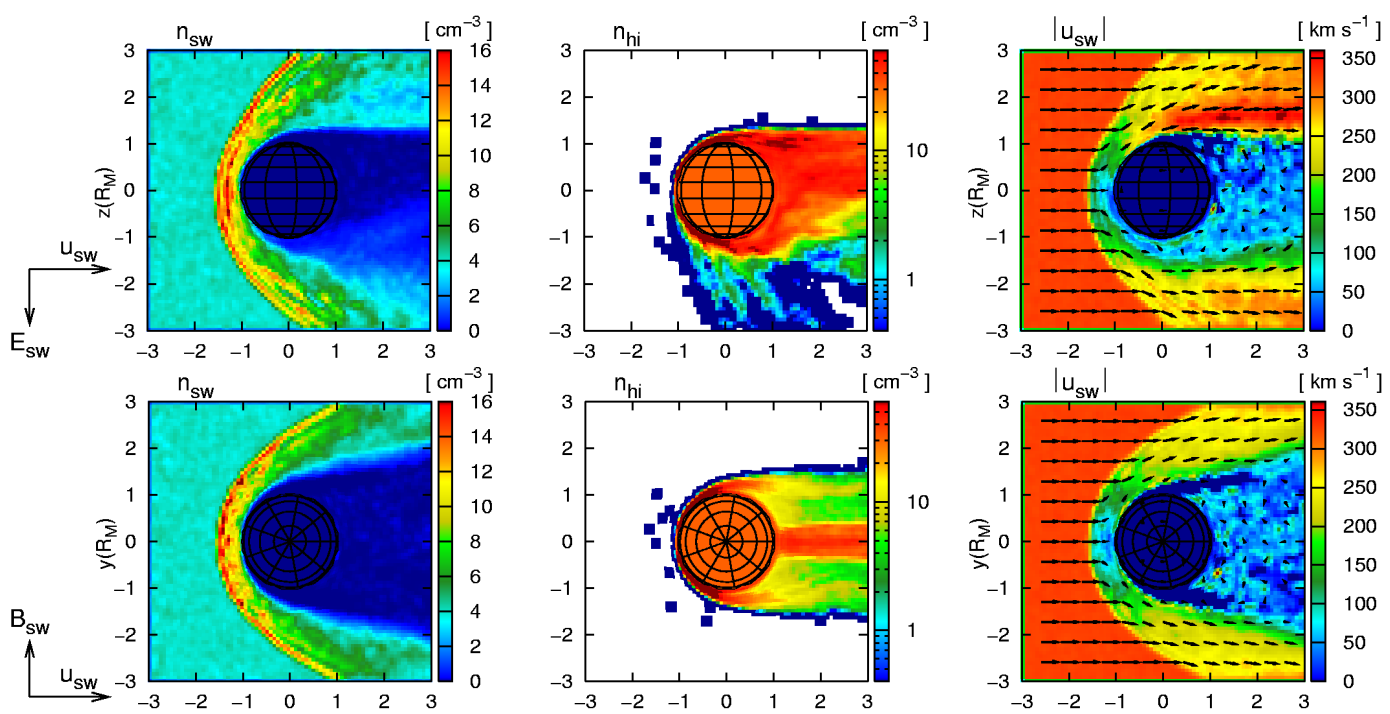

Fig. 6. Simulation results in the polar plane (top) and in the equator plane (bottom). The subfigures display from right to left the solar wind density $\left(n_{\mathrm{sw}}\right)$, the heavy ion density $\left(n_{\mathrm{hi}}\right)$ and the solar wind bulk velocity $\left(u_{\mathrm{sw}}\right)$. Besides the bow shock in front of the obstacle an further boundary the ICB can be seen behind it. At this boundary the solar wind density goes down and the heavy ion density increase sharply. On the nightside the simulation results show a plasma sheet and density rays consisting of heavy ions.
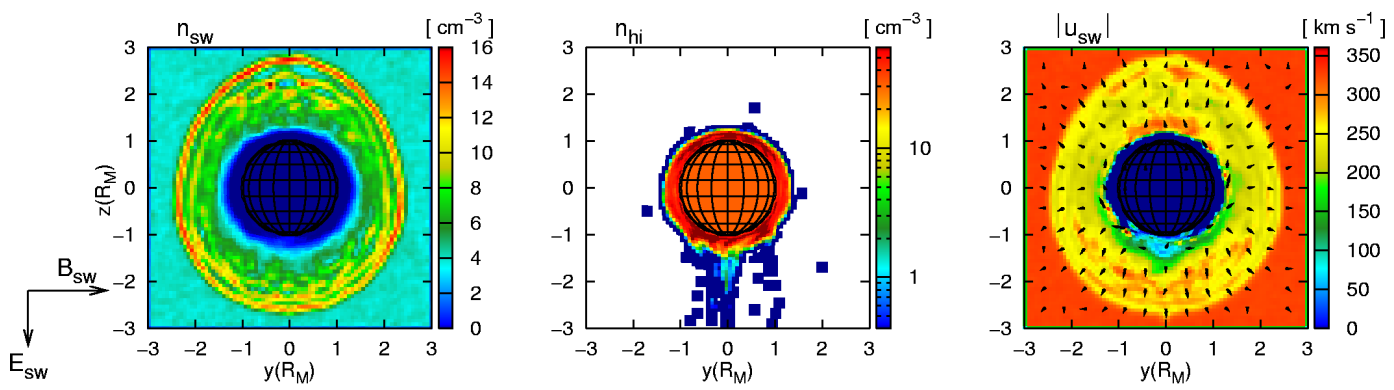

Fig. 7. Simulation results in the terminator plane. The subfigures represent the same parameters like in Fig. 6. The solar wind comes from behind the paper plane. The oval shape of the bow shock is due to the propagation of the fast magnetosonic wave which depends stronly on the direction of the IMF.

to fluctuations in the upstream solar wind which is assumed to be completely homogeneous in the simulation. The bow shock crossings are denoted by an increase of plasma density by a factor of $3-4$, which are also reproduced by the simulation model. Figure 9 also shows a good quantitative correspondence with respect to the absolut measured values.

As can be seen in Fig. 8, during the inbound pass, the electrons in the solar wind possess a temperature of about $5 \mathrm{eV}$, whereas the instrument detects an increase by a factor of 5 when passing the magnetosheath region. In contrast to this, in the simulation scenario, the temperature increases from $17 \mathrm{eV}$ to $50 \mathrm{eV}$, i.e. the jump is not as pronounced as in reality. The reason for this discrepancy may be the simplifying adiabatic describtion of the electrons in the simulations, i.e. any kind of thermal flux is suppressed. Figure 9 also shows a slight increase from $5 \mathrm{eV}$ to about $15 \mathrm{eV}$ for the inbound crossing and to about $20 \mathrm{eV}$ for the outbound pass.

At the nightside between 16:00 UT and 17:00 UT, the electron density, the thermal pressure and the proton temperature obtained from the simulation differ significantly from the values detected by the spacecraft. Since the fit of the observed density and thermal pressure displayed in Fig. 8 is meant to match the high energy part of the spectrum, it is unable to provide an adequate description of signatures obserserved in the low-energy ionospheric regime. 

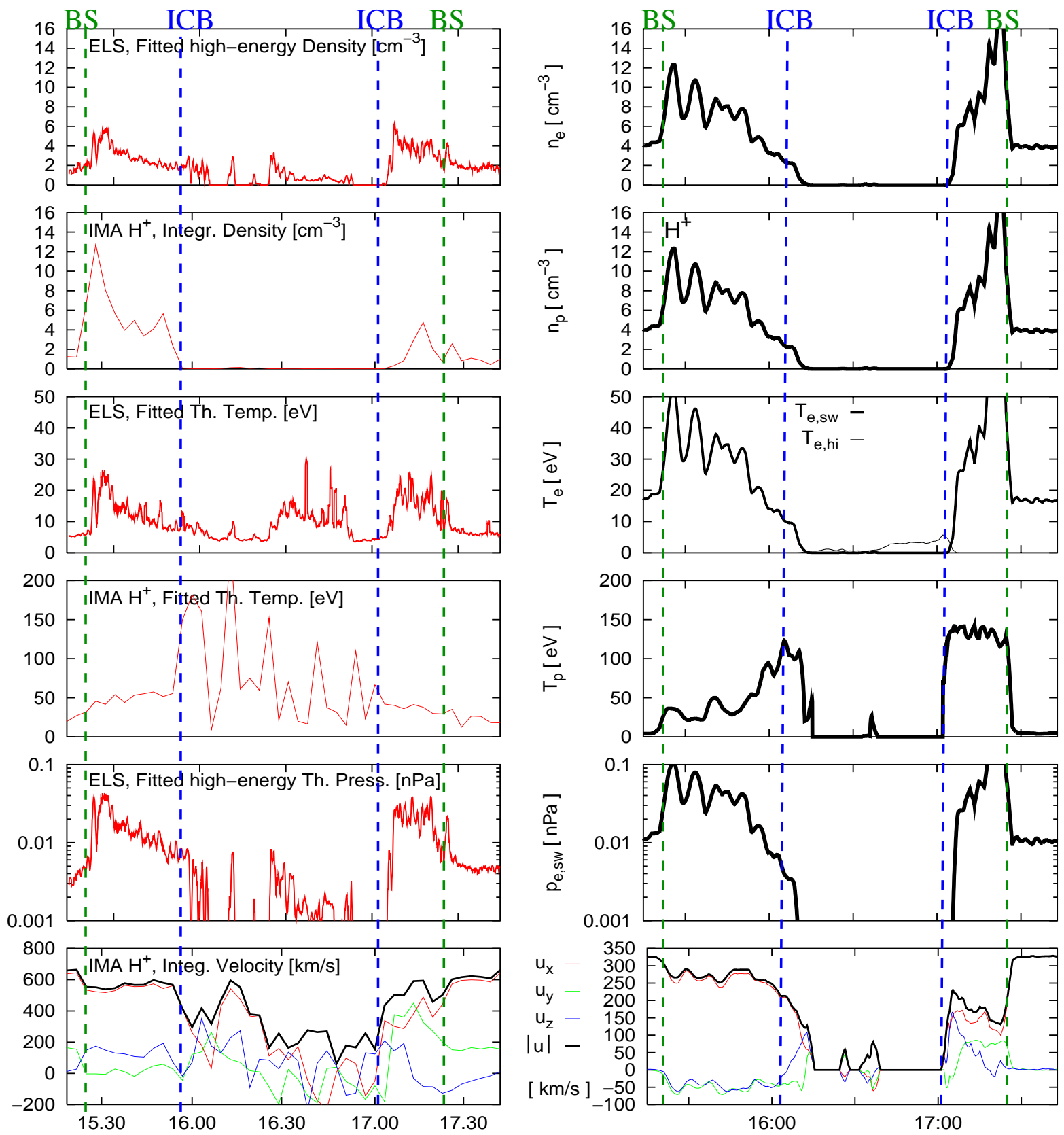

Fig. 8. Smoothed ELS und IMA data at the left hand side is represented in direct comparison with results from a hybrid simulation on the right hand side. The simulation data were extracted exactly for the MEX trajectory. Additionally positions of the bow shock and of the ICB/MPB are marked in green and blue dashed lines. Displayed are from top to bottom: the electron and proton density, as well as the temperature, the thermal electron pressure and the components as well as the total value of the proton velocity. The simulation results show a good qualitative agreement with data obtained from ASPERA-3/MEX both at the developing and at the positions of the boundaries.

The bottom panels in Figs. 8 and 9 allow a comparison of the proton velocities in the simulation with the measured data. The deceleration of the solar wind during the bow shock and magnetosheath crossings is reproduced by the simulation quite well. The decrease of proton velocity denoting the position of the ICB is detected by the spacecraft at
15:55 UT and 17:00 UT. However, while the jump positions of the proton velocity are reproduced by simulation run 1 , a significant difference can be found in the absolute values. It is likely that the velocity value measured in this region is falsified since the proton density is too small to allow an adequate averaging process. Figure 9 shows a good agreement 

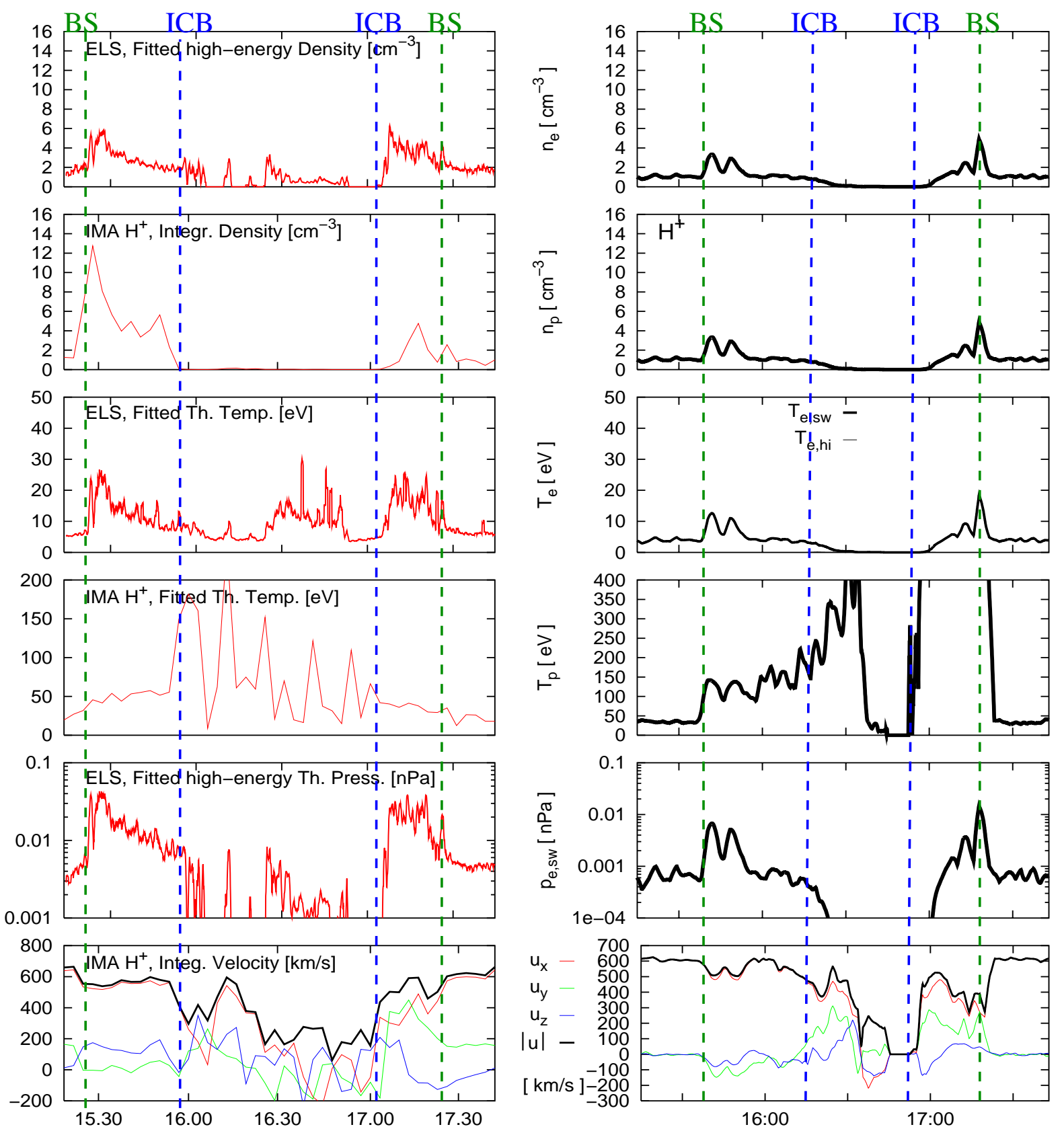

Fig. 9. Smoothed ELS und IMA data at the left hand side is represented in direct comparison with results from a second hybrid simulation on the right hand side. The positions of the bow shock and of the ICB/MPB are marked in green and blue dashed lines. Displayed are from top to bottom the same quantities as in Fig. 8. This simulation results using adaptive solar wind parameter show a good quantitative agreement with data obtained from ASPERA-3/MEX. The inbound crossings of the bow shock and the ICB/MPB appear in this simulation about 15 min later.

in the absolute values, but minor differences in the position of the boundaries.

\section{Conclusions}

In this paper, the plasma signatures detected during the Mars-Express flyby of Mars are discussed in terms of a three-dimensional hybrid simulation model, treating the electrons as a massless, charge-neutralizing fluid, whereas a 
completely kinetic approach is retained to cover ion dynamics. The Martian plasma environment is characterized by a set of sharply pronounced plasma boundaries. On the one hand, the interaction gives rise to a bow shock in front of the obstacle, whose kinetic nature is underlined by the formation of a shocklet structure. On the other hand, the solar wind is clearly separated from the ionospheric plasma by an Ion Composition Boundary. As these signatures occur in both the spacecraft data and the simulation results, the hybrid approach is capable of providing an adequate picture of the global plasma processes at Mars.

Future investigations will be necessary to investigate whether the Martian plasma environment will be affected by the multi-species nature of the ionosphere. The escape of heavy ions seemed to be primarily influenced by the direction of the convective electric field. For the simulation the convective field is assumed constant in strength and direction. In contrast to this, in the real case it can vary both in direction and magnitude on shorter time scales than a MEX periapsis crossing. The nature of these field fluctuations will be analysed in a next step using hybrid simulations by varying of the input parameters.

Acknowledgements. The authors thank D. A. Brain for several constructive discussions at the AGU Fall Meeting 2006 as well as providing values of the solar wind magnetic field by MGS. This work has been supported by the Deutsche Forschungsgemeinschaft through the grants MO 539/13-1 and MO 539/15-1.

Topical Editor I. A. Daglis thanks S. Vennerstrom and another anonymous referee for their help in evaluating this paper.

\section{References}

Acuña, M. H., Connerney, J. E. P., Wasilewski, P., Lin, R. P., Anderson, K. A., Calson, C. W., McFadden, J., Curtis, D. W., Mitchell, D., Rème, H., Mazelle, C., Sauvaud, J. A., d'Uston, C., Cros, A., Medale, J. L., Bauer, S. J., Cloutier, P., Mayhew, M., Winterhalter, D., and Ness, N. F.: Magnetic field and plasma observations at Mars: Initial results of the Mars Global Surveyor Mission, Science, 279, 1676-1680, 1998.

Bagdonat, T. and Motschmann, U.: 3D Hybrid Simulation Code Using Curvilinear Coordinates, J. Comput. Phys., 183, 470-485, 2002a.

Bagdonat, T. and Motschmann, U.: From a weak to a strong comet - 3D global hybrid simulation results, Earth, Moon and Planets, 90, 305-321, 2002b.

Bagdonat, T., Motschmann, U., Glassmeier, K.-H., and Kührt, E.: Plasma environment of comet Churyumov-Gerasimenko 3D hybrid code simulations, in: ASSL Vol. 311: The New Rosetta Targets. Observations, Simulations and Instrument Performances, edited by: Colangeli, L., Mazzotta Epifani, E., and Palumbo, P., pp. 153-155, 2004.

Barabash, S., Lundin, R., Andersson, H., Gimholt, J., Holmström, M., Norberg, O., Yamauchi, M., Asamura, K., Coates, A. J., Linder, D. R., Kataria, D. O., Curtis, C. C., Hsieh, K. C., Fedorov, B. R. S. A., Grigoriev, A., Budnik, E., Grande, M., Carter, M., Reading, D. H., Koskinen, H., Kallio, E., Riihelä, P., Sälles, T.,
Kozyra, J., Krupp, N., Livi, S., Woch, J., Luhmann, J., McKennaLawlor, S., Orsini, S., Cerulli-Irelli, R., Mura, A., Roelof, A. M. E., Williams, D., Sauvaud, J.-A., Winningham, J.-J. T. D., Frahm, R., Scherrer, J., Wurz, J. S. P., and Bochsler, P.: The Analyzer of space plasmas and energetic atoms (ASPERA-3) for the European Mars Express mission, Space Sci. Rev., 126, 113-164, doi:10.1007/s11214-006-9124-8, 2006.

Bößwetter, A., Bagdonat, T., Motschmann, U., and Sauer, K.: Plasma boundaries at Mars: a 3-D simulation study, Ann. Geophys., 22, 4363-4379, 2004, http://www.ann-geophys.net/22/4363/2004/.

Brace, L. H., Theis, R. F., Mayr, H. G., Curtis, S. A., and Luhmann, J. G.: Holes in the nightside ionosphere of Venus, J. Geophys. Res., 87, 199-211, 1982.

Brecht, S. H.: Hybrid simulations of the magnetic topology of Mars, J. Geophys. Res., 102, 4743-4750, 1997.

Breus, T. K., Krymskii, A. M., Lundin, R., Dubinin, E. M., Luhmann, J. G., Yeroshenko, Y. G., Barabash, S. V., Mitnitskii, V. Y., Pissarenko, N. F., and Styashkin, V. A.: The solar wind interaction with Mars: consideration of Phobos-2 mission observations of an ion composition boundary on the dayside, J. Geophys. Res., 96, 11 165-11 174, 1991.

Fedorov, A., Budnik, E., Sauvaud, J.-A., Mazelle, C., Barabash, S., Lundin, R., Acuña, M., Holmström, M., Grigoriev, A., Yamauchi, M., Andersson, H., Thocaven, J.-J., Winningham, D., Frahm, R., Sharber, J. R., Scherrer, J., Coates, A. J., Linder, D. R., Kataria, D. O., Kallio, E., Koskinen, H., Säles, T., Riihelä, P., Schmidt, W., Kozyra, J., Luhmann, J., Roelof, E., Williams, D., Livi, S., Curtis, C. C., Hsieh, K. C., Sandel, B. R., Grande, M., Carter, M., McKenna-Lawler, S., Orsini, S., Cerulli-Irelli, R., Maggi, M., Wurz, P., Bochsler, P., Krupp, N., Woch, J., Fränz, M., Asamura, K., and Dierker, C.: Structure of the martian wake, Icarus, 182, 329-336, doi:10.1016/j.icarus.2005.09.021, 2006.

Fränz, M., Roussos, E., Dubinin, E., Martinecz, C., Woch, J., Winningham, J., Frahm, R., Fedorov, A., Coates, A. J., Barabash, S., and Lundin, R.: Plasma Moments in the environment of Mars, Space Sci. Rev., 126, 165-207, doi:10.1007/s11214-006-91159, 2006a.

Fränz, M., Winningham, J. D., Dubinin, E., Roussos, E., Woch, J., Barabash, S., Lundin, R., Holmström, M., Andersson, H., Yamauchi, M., Grigoriev, A., Frahm, R. A., Sharber, J. R., Scherrer, J. R., Coates, A. J., Soobiah, Y., Linder, D. R., Kataria, D. O., Kallio, E., Säles, T., Riihelä, P., Schmidt, W., Koskinen, H. E. J., Kozyra, J., Luhmann, J., Roelof, E., Williams, D., Livi, S., Curtis, C. C., Hsieh, K. C., Sandel, B. R., Grande, M., Carter, M., Sauvaud, J.-A., Fedorov, A., Thocaven, J.-J., McKenna-Lawler, S., Orsini, S., Cerulli-Irelli, R., Maggi, M., Wurz, P., Bochsler, P., Krupp, N., Asamura, K., and Dierker, C.: Plasma intrusion above Mars crustal fields - Mars Express ASPERA-3 observations, Icarus, 182, 406-412, doi:10.1016/j.icarus.2005.11.016, 2006b.

Hanson, W. B. and Mantas, G. P.: Viking electron temperature Measurements: Evidence for a magnetic field in the Martian ionosphere, J. Geophys. Res., 93, 7538-7544, 1988.

Israelevich, P. L., Gombosi, T. I., Ershkovich, A. I., DeZeeuw, D. L., Neubauer, F. M., and Powell, K. G.: The induced magnetosphere of comet Halley. 4. Comparison of in situ observations and numerical simulations, J. Geophys. Res., 104, 28309 $28319,1999$. 
Kallio, E. and Janhunen, P.: Ion escape from Mars in a quasi-neutral hybrid model, J. Geophys. Res., 107, 1035, doi:10.1029/2001JA000090, 2002.

Kallio, E., Fedorov, A., Budnik, E., Säles, T., Janhunen, P., Schmidt, W., Koskinen, H., Riihelä, P., Barabash, S., Lundin, R., Holmström, M., Gunell, H., Brinkfeldt, K., Futaana, Y., Andersson, H., Yamauchi, M., Grigoriev, A., Sauvaud, J.-A., Thocaven, J.J., Winningham, J. D., Frahm, R. A., Sharber, J. R., Scherrer, J. R., Coates, A. J., Linder, D. R., Kataria, D. O., Kozyra, J., Luhmann, J. G., Roelof, E., Williams, D., Livi, S., Curtis, C. C., Hsieh, K. C., Sandel, B. R., Grande, M., Carter, M., McKennaLawler, S., Orsini, S., Cerulli-Irelli, R., Maggi, M., Wurz, P., Bochsler, P., Krupp, N., Woch, J., Fränz, M., Asamura, K., and Dierker, C.: Ion escape at Mars: Comparison of a 3-D hybrid simulation with Mars Express IMA/ASPERA-3 measurements, Icarus, 182, 350-359, 2006.

Kanao, M., Terada, N., Yamazaki, A., Yoshikawa, I., Abe, T., and Nakamura, M.: Effect of the motional elecric field on the Venus nightside ionopause, J. Geophys. Res., 111, A10, doi:10.1029/ 2005JA0112903, 2006.

Lichtenegger, H. and Dubinin, E.: Model calculation of the planetary ion distribution in the Martian tail, Earth Planets Space, 50, 445-452, 1998.

Liu, Y., Nagy, A. F., Groth, P. T., DeZeeuw, D. L., and Gombosi, T. I.: 3D Multi-fluid MHD Studies of the Solar Wind Interaction with Mars, Geophys. Res. Lett., 26, 2689-2692, 1999.

Luhmann, J. G.: The Magnetopause Counterpart at the Weakly Magnetized Planets: The Ionopause, in: Physics of the Magnetopause, vol. 90 of Geophysical Monograph, pp. 71-79, American Geophysical Union, 1995.

Lundin, R., Borg, H., Hultqvist, B., Zakharov, A., and Pellinen, R.: First measurements of the ionospheric plasma escape from Mars, Nature, 341, 609-612, doi:10.1038/341609a0, 1989.

Lundin, R., Zakharov, A., Pellinen, R., Barabash, S. W., Borg, H., Dubinin, E. M., Hultquist, B., Koskinen, H., Liede, I., and Pissarenko, N.: ASPERA/Phobos measurements of the ion outflow from the Martian ionosphere, Geophys. Res. Lett., 17, 873-876, 1990.

Mazelle, C., Rème, H., Sauvaud, J. A., d'Uston, C., Carlson, C. W., Anderson, K. A., Curtis, D. W., Lin, R. P., Korth, A., Mendis, D. A., Neubauer, F. M., Glassmeier, K. H., and Raeder, J.: Analysis of suprathermal electron properties at the magnetic pile-up boundary of comet P/Halley, Geophys. Res. Lett., 16, 10351038, 1989.

Mitchell, D. L., Lin, R. P., Rème, H., Crider, D. H., Cloutier, P. A., Connerney, J. E. P., Acuña, M. H., and Ness, N. F.: Oxygen Auger electrons observed in Mars' ionosphere, Geophys. Res. Lett., 27, 1871-1874, 2000.

Modolo, R., Chanteur, G. M., Dubinin, E., and Matthews, A. P.: Influence of the solar EUV flux on the Martian plasma environment, Ann. Geophys., 23, 433-444, 2005,

http://www.ann-geophys.net/23/433/2005/.

Motschmann, U. and Kührt, E.: Interaction of the Solar Wind with Weak Obstacles: Hybrid Simulations for Weakly Active Comets and for Mars, Space Sci. Rev., 122, 197-207, doi: 10.1007/s11214-006-6218-2, 2006.

Nagy, A. F., Winterhalter, D., Sauer, K., Cravens, T. E., Brecht, S., Mazelle, C., Crider, D., Kallio, E., Zakharov, A., Dubinin, E., Verigin, M., Kotova, G., Axford, W. I., Bertucci, C., and
Trotignon, J. G.: The plasma Environment of Mars, Space Sci. Rev., 111, 33-114, doi:10.1023/B:SPAC.0000032718.47512.92, 2004.

Omidi, N. and Winske, D.: Steepening of kinetic magnetosonic waves into shocklets: Simulations and consequences for planetary shocks and comets, J. Geophys. Res., 95, 2281-2300, 1990.

Penz, T., Erkaev, N. V., Biernat, H. K., Lammer, H., Amerstorfer, U. V., Gunell, H., Kallio, E., Barabash, S., Orsini, S., Milillo, A., and Baumjohann, W.: Ion loss on Mars caused by the KelvinHelmholtz instability, Planet. Space Sci., 52, 1157-1167, doi: 10.1016/j.pss.2004.06.001, 2004.

Rème, H., Mazelle, C., Sauvaud, J. A., d'Uston, C., Froment, F., Lin, R. P., Anderson, K. A., Carlson, C. W., Larson, D. E., Korth, A., Chaizy, P., and Mendis, D. A.: Electron Plasma Environment at Comet Grigg-Skjellerup: General Observations and Comparison With the Environment at Comet Halley, J. Geophys. Res., 98, 20 965-20976, 1993.

Riedler, W., Möhlmann, D., Oraevsky, V. N., Schwingenschuh, K., Yeroshenko, Y., Rustenbach, J., Aydogar, O., Berghofer, G., Lichtenegger, H., Delva, M., Schelch, G., Pirsch, K., Fremuth, G., Steller, M., Arnold, H., Raditsch, T., Auster, U., Fornacon, K.-H., Schenk, H. J., Michaelis, H., Motschmann, U., Roatsch, T., Sauer, K., Schrer, R., Kurths, J., Lenners, D., Linthe, J., Kobzev, V., Styashkin, V., Achache, J., Slavin, J., Luhmann, J. G., and Russell, C. T.: Magnetic fields near Mars: first results, Nature, 341, 604-607, 1989.

Riedler, W., Schwingenschuh, K., Lichtenegger, H., Möhlmann, D., Rustenbach, J., Weroshenko, Y., Achache, J., Slavin, J., Luhmann, J. G., and Russell, C. T.: Interaction of the solar wind with the planet Mars: Phobos-2 magnetic field observations, Planet. Space Sci., 39, 75-81, 1991.

Rosenbauer, H., Shutte, N., Apáthy, I., Galeev, A., Gringauz, K., Grünwaldt, H., Hemmerich, P., Jockers, K., Király, P., Kotova, G., Livi, S., Marsch, E., Richter, A., Riedler, W., Remizov, T., Schwenn, R., Schwingenschuh, K., Steller, M., Szegö, K., Verigin, M., and Witte, M.: Ions of Martian origin and plasma sheet in the Martian magnetosphere/ initial results of the TAUS experiment, Nature, 341, 612-614, 1989.

Sauer, K., Roatsch, T., Motschmann, U., Schwingenschuh, K., Lundin, R., Rosenbauer, H., and Livi, S.: Observations of Plasma Boundaries and Phenomena Around Mars with Phobos 2, J. Geophys. Res., 97, 6227-6233, 1992.

Sauer, K., Bogdanov, A., and Baumgärtel, K.: Evidence of an ion composition boundary (protonopause) in bi-ion fluid simulations of solar wind mass loading, Geophys. Res. Lett., 21, 2255-2258, 1994.

Shimazu, H.: Three-dimensional hybrid simulation of magnetized plasma flow around an obstacle, Earth Planets Space, 51, 383393, 1999.

Shimazu, H.: Three-dimensional hybrid simulation of solar wind interaction with unmagnetized planets, J. Geophys. Res., 106, 8333-8342, 2001.

Simon, S., Bagdonat, T., Motschmann, U., and Glassmeier, K.-H.: Plasma environment of magnetized asteroids: a 3-D hybrid simulation study, Ann. Geophys., 24, 407-414, 2006a.

Simon, S., Boesswetter, A., Bagdonat, T., Motschmann, U., and Glassmeier, K.-H.: Plasma environment of Titan: a 3-D hybrid simulation study, Ann. Geophys., 24, 1113-1135, 2006b.

Simon, S., Boesswetter, A., Bagdonat, T., and Motschmann, U.: 
A comparative 3-D hybrid simulation study of Mars and Titan, Ann. Geophys., 25, 99-115, 2007, http://www.ann-geophys.net/25/99/2007/.

Spreiter, J. R. and Stahara, S. S.: Computer modeling of the solar wind interaction with Venus and Mars, in: Venus and Mars: Atmospheres, Ionospheres, and Solar Wind interactions, vol. 66 of Geophysical Monograph, pp. 345-383, American Geophysical Union, 1992.

Terada, N., Machida, S., and Shinagawa, H.: Global hybrid simulation of the Kelvin-Helmholtz instability at the Venus ionopause, J. Geophys. Res., 107, 30-1, doi:10.1029/2001JA009224, 2002.

Vennerstrom, S., Olsen, N., Purucker, M., Acuña, M. H., and Cain, J. C.: The magnetic field in the pile-up region at Mars, and its variation with the solar wind, Geophys. Res. Lett., 30, 1369, doi:10.1029/2003GL016883, 2003.
Vignes, D., Mazelle, C., Rème, H., Acuña, M. H., Connerney, J. E. P., Lin, R. P., Mitchell, D. L., Cloutier, P., Crider, D. H., and Ness, N. F.: The Solar Wind interaction with Mars: locations and shapes of the Bow Shock and the observations of the MAG/ER experiment onboard Mars Global Surveyor, Geophys. Res. Lett., 27, 49-52, 2000.

Wahlund, J.-E., Boström, R., Gustafsson, G. A. G. D., Kurth, W. S., Pedersen, A., Averkamp, T. F., Hospodarsky, G. B., Persoon, A. M., Canu, P., Neubauer, F. M., Dougherty, M. K., Eriksson, A. I., Morooka, M. W., Gill, R., André, M., Eliasson, L., and Mueller-Wodarg, I.: Cassini Measurements of Cold Plasma in the Ionosphere of Titan, Science, 308, 986-989, 2005.

Zhang, T. L., Luhmann, J. G., and Russell, C. T.: The magnetic barrier at Venus, J. Geophys. Res., 96, 11 145-11 153, 1991. 